\title{
Monodromy in the water molecule
}

\author{
Nikolai F. Zobov ${ }^{a c}$, Sergei V. Shirin ${ }^{a c}$, Oleg L. Polyansky ${ }^{b}$, \\ Jonathan Tennyson ${ }^{a}$,*, Pierre-François Coheur ${ }^{\mathrm{d}}$, \\ Peter F. Bernath ${ }^{\mathrm{e}}$, Michel Carleer ${ }^{\mathrm{d}}$, Reginald Colin ${ }^{\mathrm{d}}$

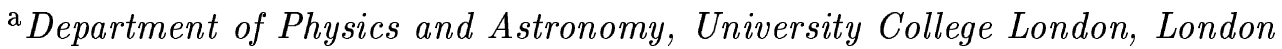 \\ WC1E 6BT, UK \\ ${ }^{\mathrm{b}}$ Arbeitsgruppe Chemieinformationssysteme, Albert-Einstein-Allee 47, Ulm \\ University, Ulm, Germany \\ ${ }^{\mathrm{c}}$ Permanent address: Institute of Applied Physics, Russian Academy of Science, \\ Uljanov Street 46, Nizhnii Novgorod, Russia 603950. \\ ${ }^{\mathrm{d}}$ Université Libre de Bruxelles, Service de Chimie Quantique et Photophysique, 50 \\ Av. F.D. Roosevelt, B-1050 Bruxelles, Belgium \\ e Department of Chemistry, University of Waterloo, Waterloo, ON Canada N2L \\ $3 G 1$ and Department of Chemistry, University of Arizona, Tucson, AZ, 85721
}

\begin{abstract}
The change in the energy level structure of a bent molecule as it starts to sample linear geometries has been discussed in terms of monodromy found in simple two dimensional model problems. Infrared spectra of hot $(T \sim 3000 \mathrm{~K})$ water are analysed for transitions involving states with high bending excitation which can sample linear $\mathrm{HOH}$ geometries. 134 new experimental energy levels are determined for bending states with $5 \leq v_{2} \leq 9$ including band origins for $7 \nu_{2}, 8 \nu_{2}$ and $9 \nu_{2}$. Plots of those levels with $J=K_{a}$ show the characteristic rearrangement of energy levels predicted for a system displaying quantum monodromy. Quantum monodromy for water occurs about the $\nu_{2}=7, J=0$ level. The barrier to linearity in $\mathrm{H}_{2}{ }^{16} \mathrm{O}$ is estimated to lie at $11114 \pm 5 \mathrm{~cm}^{-1}$.
\end{abstract}

\section{INTRODUCTION}

Monodromy, which means once round, is a concept arising from classical mechanics [1-3]. Monodromy in quantum mechanical systems implies the absence

\footnotetext{
* Author to whom correspondence should be addressed.

Email address: j.tennyson@ucl.ac.uk (Jonathan Tennyson).
} 
of a single, smoothly-varying set of quantum numbers with which to characterise the system. A proto-typical example of a problem displaying monodromy is the energy levels of a Mexican-hat shaped potential (also sometimes likened to the shape at the bottom of a champagne bottle). In these potentials one set of quantum numbers, characterised by free angular motion around the rim of the hat and the perpendicular vibration, exists at low energy. Once the energy levels reach the peak of the hat, the monodromy point, the energy levels restructure and require a new set of quantum numbers.

As noted by Child et al. [4] the rotation-vibration energy levels of bent molecules display monodromy when they sample linear geometries. Child et al. illustrate this behaviour with calculated energy levels for water. Similar conclusions have been drawn from a theoretical analyses of the HCP molecule [5] and the isomerising LiNC/LiCN system [6]. Despite many experimental studies on bent molecules with low barriers to linearity (see for example, B. Winnewisser's review [7]), it would appear that thus far published spectroscopic studies of these quasi-linear systems have either been for problems for which the barrier to linearity is so low that the energy level structure characteristic of a bent system does not really exist, or where the barrier to linearity is so high that insufficient excitation has been achieved to probe levels that cross it. Very recently, Winnewisser et al. [8] have returned to the study of the pure rotational spectrum of the NCNCS molecule, which also clearly displays monodromy. Conversely a study of levels about the barrier to isomeration in the $\mathrm{HCN} / \mathrm{HNC}$ system showed the absence of monodromy meaning that all bending modes in this system could be assigned on the basis of a single, global bending quantum number [9].

The precise height of the barrier to linear $\mathrm{HOH}$ geometries in water has been the subject of a series of theoretical studies [10-13] and is thought to be close to $11100 \mathrm{~cm}^{-1}[12,13]$. The eighth bending overtone, $9 \nu_{2}$, and possibly the seventh bending overtone, $8 \nu_{2}$, are predicted to be the lowest bending states with sufficient energy to sample linear geometries $[4,14]$. In this paper we report analysis of infrared spectra of hot water which yield transitions involving levels of $v_{2}=9$ for the first time. Plots of these energy levels and appropriate ones for lower bending overtones show the clear signature one would expect for a system displaying monodromy.

\section{METHOD}

The results reported in this letter are based on the analysis of two complementary spectra of hot water. The first spectrum is the recently reported laboratory emission spectrum of water vapour at about $3000 \mathrm{~K}$ recorded using an oxy-acetylene torch by Coheur et al. [15]. This spectrum spans most of the in- 
frared, although only the regions with pure rotational transitions and bending transitions will concern us here. The second spectrum analysed is an absorption spectrum recorded in a sunspot spanning the $722-1011 \mathrm{~cm}^{-1}$ region [16]. This spectrum is dominated by absorptions by water which is thought to have a temperature of $3200 \mathrm{~K}$. The sunspot spectrum, in particular, has been available and partially assigned for some time [17, 18].

These spectra contain information on the pure rotational transitions, which lie mostly below $1000 \mathrm{~cm}^{-1}$, and bending transitions, which lie mostly in the $1000-2000 \mathrm{~cm}^{-1}$ region. In both cases it is not possible to analyse transitions involving states of high bending excitation without also considering similar transitions involving states of lower bending excitation. Previous studies on hot water have characterised the levels for $5 \nu_{2}$ [19] and $6 \nu_{2}[20,21]$. Our recent analysis of the torch and sunspot spectra [15] extended the results for $6 \nu_{2}$ and identified levels for both $7 \nu_{2}$ and $8 \nu_{2}$; in particular the 10 levels (tentatively) ascribed to $8 \nu_{2}$ were the first assigned to this state.

New assignments were made by comparison with predictions made from variational nuclear motion calculations. These calculations were based on a spectroscopic potential energy surface which was specifically tuned to treat the bending motions. Starting from the best available ab initio potential [22], the surface was morphed [23] to fit the experimentally observed bending states for $n \nu_{2}$ with $n=0-7$ with total rotational quantum number $J$ equal to $0,2,5$ and 10. This new potential reproduced these bending level with a standard deviation of $0.05 \mathrm{~cm}^{-1}$, although it performed much worse for states with stretching excitation. This is a significant improvement for bending levels on our previous best spectroscopically-determined potential [23], which gave residuals of up to $1 \mathrm{~cm}^{-1}$ for certain levels in the $7 \nu_{2}$ and $8 \nu_{2}$ vibrational states. This fit finds of the lowest $\mathrm{HOH}$ linear geometry for $R_{\mathrm{OH}}=0.93329 \AA$ at a point $11114 \mathrm{~cm}^{-1}$ above the minimum of the potential energy surface, for which $R_{\mathrm{OH}}=0.95785$ $\AA$. A conservative estimate for the error in the barrier height is $5 \mathrm{~cm}^{-1}$.

\section{RESULTS}

The new line assignments made as part of this work lead to 134 additional experimental energy levels being characterised: 25 for $5 \nu_{2}, 45$ for $6 \nu_{2}, 25$ for $7 \nu_{2}$, 25 for $8 \nu_{2}$ and, for the first time, 14 for $9 \nu_{2}$, see Table 1. The errors in these energy levels are about $0.05 \mathrm{~cm}^{-1}$, which is comparatively large for a spectroscopic study. However both spectra were obtained at very high temperatures so show significant Doppler broadening, furthermore the torch spectrum was recorded at atmospheric pressure and the lines are all significantly pressure broadened, see [15], and the sunspot spectrum was recorded at a resolution

of only $0.02 \mathrm{~cm}^{-1}$. Finally several of the transitions assigned in this study are 
blended.

Our analysis gives the first experimental values for the band origins of $7 \nu_{2}, 8 \nu_{2}$ and $9 \nu_{2}$ at $10086.0 \pm 0.1,11254.0 \pm 0.2$ and $12533.7 \pm 0.3 \mathrm{~cm}^{-1}$, respectively. The last two states thus lie at energies above the linear $\mathrm{HOH}$ geometry.

For the higher states there was usually only one strong transition associated with each level in the experimental spectra. This means that many levels, and in particular all those with $J=K_{a}$ for $7 \nu_{2}, 8 \nu_{2}$ and $9 \nu_{2}$, have not been confirmed by combination differences.

The most straightforward way of detecting monodromy in the bending spectrum of water is to analyse the patterns given by rotation-vibration levels which differ in the bending quantum number, $v_{2}$, and have rotational quantum numbers characterised by $J=K_{a} . K_{a}$ is the bent molecule, ie asymmetric top, quantum number which approximately characterises motion along the $\mathrm{A}$ axis for which the moment of inertia tends to zero as the molecule become linear. States with $J=K_{a}$ are the quantum mechanically allowed states which come closest to undergoing free rotation about the monodromy point. Conversely the bending series corresponds to the perpendicular motion. As shown previously, plots of these levels show the characteristic pattern expected for a system for which the level structure is rearranging about a monodromy point, see Figure 1 of ref. [4].

A plot of all the experimentally-determined energy levels taken from this work and previous studies [19-21, 25] with $v_{2} \geq 5$ and $J=K_{a} \leq 9$ is given in fig. 1 . To make the monodromy structure transparent in this figure, all levels with $J=K_{a} \neq 0$ have been plotted twice, once for positive $K_{a}$ and once for negative $K_{a}$.

Figure 1 shows the characteristic change in energy level structure one associates with monodromy. Thus, for example, the levels for $5 \nu_{2}$ and $6 \nu_{2}$ form a smooth parabola through the $J=0$ level, whereas those for $8 \nu_{2}$ and $9 \nu_{2}$ have a clear discontinuity in the slope about the $K_{a}=0$ level but are well represented by a straight line either side of this point. The levels of $7 \nu_{2}$ seem to be intermediate between these two behaviours which can be characterised as those of a bent and a (quasi-)linear molecule, respectively.

Table 1: Energies of the newly assigned levels of $\mathrm{H}_{2}{ }^{16} \mathrm{O}$ obtained by analysing the laboratory torch spectrum of Coheur et al. [15] or the sunspot spectrum of Wallace et al. [16].

\begin{tabular}{lllll}
\hline$J$ & $K_{a}$ & $K_{c}$ & $v_{1} v_{2} v_{3}$ & Energy \\
\hline
\end{tabular}




\begin{tabular}{|c|c|c|c|c|}
\hline 2 & 1 & 1 & 050 & 7682.89 \\
\hline 4 & 0 & 4 & 050 & 7770.40 \\
\hline 4 & 2 & 2 & 050 & $8002.56^{a}$ \\
\hline 5 & 4 & 2 & 050 & 8608.10 \\
\hline 6 & 1 & 6 & 050 & $8024.94^{a}$ \\
\hline 6 & 5 & 1 & 050 & 9054.36 \\
\hline 7 & 1 & 7 & 050 & $8162.64^{a}$ \\
\hline 8 & 0 & 8 & 050 & 8307.65 \\
\hline 8 & 1 & 7 & 050 & $8529.70^{a}$ \\
\hline 9 & 1 & 8 & 050 & $8747.25^{a}$ \\
\hline 9 & 5 & 4 & 050 & $9645.93^{a}$ \\
\hline 9 & 9 & 1 & 050 & 10944.10 \\
\hline 9 & 9 & 0 & 050 & 10944.10 \\
\hline 10 & 4 & 7 & 050 & $9570.46^{a}$ \\
\hline 10 & 5 & 5 & 050 & $9869.81^{a}$ \\
\hline 11 & 2 & 9 & 050 & $9430.25^{a}$ \\
\hline 11 & 8 & 3 & 050 & 11139.14 \\
\hline 12 & 0 & 12 & 050 & $9120.19^{a}$ \\
\hline 12 & 8 & 5 & 050 & 11426.92 \\
\hline 13 & 7 & 6 & 050 & 11404.91 \\
\hline 13 & 8 & 5 & 050 & 11736.39 \\
\hline 14 & 0 & 14 & 050 & $9632.85^{a}$ \\
\hline 14 & 7 & 8 & 050 & $11737.65^{a}$ \\
\hline 15 & 4 & 11 & 050 & $11141.74^{a}$ \\
\hline 15 & 5 & 10 & 050 & 11409.57 \\
\hline 1 & 0 & 1 & 060 & $8893.52^{a}$ \\
\hline 2 & 1 & 2 & 060 & $9038.76^{a}$ \\
\hline 2 & 1 & 1 & 060 & $9058.37^{a}$ \\
\hline
\end{tabular}




\begin{tabular}{|c|c|c|c|c|}
\hline & 0 & 3 & 060 & $9008.72^{a}$ \\
\hline & 1 & & 060 & 9099.65 \\
\hline & & 2 & 060 & $9138.72^{a}$ \\
\hline & 2 & 2 & 060 & 9343.04 \\
\hline & & 4 & 060 & $9098.94^{a}$ \\
\hline 4 & 1 & 4 & 060 & $9180.36^{a}$ \\
\hline & & & 060 & 9245.18 \\
\hline & 3 & 1 & 060 & 9725.86 \\
\hline & 0 & 5 & 060 & $9209.63^{a}$ \\
\hline$r$ & 1 & 5 & 060 & $9280.75^{a}$ \\
\hline & 1 & 4 & 060 & $9377.06^{a}$ \\
\hline 5 & 3 & 3 & 060 & $9846.72^{a}$ \\
\hline & 3 & 2 & 060 & 9847.10 \\
\hline$c$ & 0 & 6 & 060 & 9339.23 \\
\hline 6 & 3 & 3 & 060 & 9992.54 \\
\hline 6 & 4 & 2 & 060 & $10316.98^{a}$ \\
\hline 6 & 5 & 2 & 060 & 10666.55 \\
\hline 6 & 5 & 1 & 060 & 10666.55 \\
\hline 7 & 0 & 7 & 060 & $9487.55^{a}$ \\
\hline 7 & 1 & 7 & 060 & $9539.20^{a}$ \\
\hline 7 & 1 & 6 & 060 & $9714.47^{a}$ \\
\hline 7 & 2 & 6 & 060 & $9861.56^{a}$ \\
\hline 1 & 2 & 5 & 060 & $9888.80^{a}$ \\
\hline 7 & 3 & 5 & 060 & 10158.51 \\
\hline 0 & 0 & 8 & 060 & $9653.84^{a}$ \\
\hline 8 & 1 & 8 & 060 & $9696.93^{a}$ \\
\hline 8 & 1 & 7 & 060 & $9918.13^{a}$ \\
\hline & 2 & 6 & 060 & $10088.40^{a}$ \\
\hline & 8 & 1 & 060 & 12109.38 \\
\hline
\end{tabular}




\begin{tabular}{|c|c|c|c|c|}
\hline 8 & 8 & 0 & 060 & 12109.38 \\
\hline 9 & 0 & 9 & 060 & $9837.79^{a}$ \\
\hline 9 & 1 & 9 & 060 & $9873.56^{a}$ \\
\hline 9 & 1 & 8 & 060 & $10143.61^{a}$ \\
\hline 9 & 2 & 8 & 060 & $10254.77^{a}$ \\
\hline 9 & 2 & 7 & 060 & $10312.11^{a}$ \\
\hline 9 & 5 & 4 & 060 & 11250.40 \\
\hline 10 & 0 & 10 & 060 & $10039.60^{a}$ \\
\hline 10 & 1 & 9 & 060 & 10390.02 \\
\hline 10 & 2 & 8 & 060 & 10558.58 \\
\hline 10 & 5 & 6 & 060 & 11491.06 \\
\hline 11 & 1 & 10 & 060 & $10656.35^{a}$ \\
\hline 12 & 0 & 12 & 060 & 10497.84 \\
\hline 0 & 0 & 0 & 070 & 10086.04 \\
\hline 1 & 0 & 1 & 070 & 10109.15 \\
\hline 4 & 4 & 1 & 070 & 11619.13 \\
\hline 5 & 0 & 5 & 070 & $10427.99^{a}$ \\
\hline 5 & 3 & 2 & 070 & 11354.67 \\
\hline 5 & 4 & 1 & 070 & 11741.89 \\
\hline 5 & 5 & 0 & 070 & $12138.84^{a}$ \\
\hline 6 & 0 & 6 & 070 & $10562.57^{a}$ \\
\hline 6 & 4 & 3 & 070 & 11888.16 \\
\hline 6 & 5 & 2 & 070 & 12286.11 \\
\hline 6 & 6 & 1 & 070 & 12686.74 \\
\hline 7 & 0 & 7 & 070 & 10718.86 \\
\hline 7 & 2 & 5 & 070 & 11296.03 \\
\hline 7 & 4 & 3 & 070 & 12061.42 \\
\hline 7 & 5 & 2 & 070 & $12456.44^{a}$ \\
\hline
\end{tabular}




\begin{tabular}{|c|c|c|c|c|}
\hline & 7 & 1 & 070 & 13245.19 \\
\hline & 7 & 0 & 070 & 13245.19 \\
\hline & 0 & 8 & 070 & 10896.61 \\
\hline & 2 & 6 & 070 & 11481.59 \\
\hline & & 9 & 070 & $11097.10^{a}$ \\
\hline & 1 & 11 & 070 & 11652.06 \\
\hline & 4 & 7 & 070 & 12935.73 \\
\hline & 1 & 12 & 070 & $11902.75^{a}$ \\
\hline & 1 & 13 & 070 & 12180.31 \\
\hline & 1 & 12 & 070 & $12673.08^{a}$ \\
\hline & 0 & 0 & 080 & $11253.99^{b}$ \\
\hline & 0 & 1 & 080 & 11277.12 \\
\hline & 1 & 1 & 080 & $11668.90^{b}$ \\
\hline & 1 & 0 & 080 & 11676.06 \\
\hline & 0 & 2 & 080 & $11322.97^{a}$ \\
\hline & 1 & 2 & 080 & $11709.23^{a}$ \\
\hline & 2 & 1 & 080 & 12219.47 \\
\hline & 3 & 0 & 080 & 12660.74 \\
\hline 4 & 2 & 3 & 080 & $12318.24^{b}$ \\
\hline & 3 & 2 & 080 & 12760.72 \\
\hline & 4 & 1 & 080 & 13203.76 \\
\hline & 0 & 5 & 080 & 11601.29 \\
\hline 5 & 1 & 4 & 080 & 12061.30 \\
\hline 5 & 2 & 3 & 080 & 12427.19 \\
\hline 5 & 3 & 2 & 080 & $12877.59^{b}$ \\
\hline & 5 & 0 & 080 & 13771.83 \\
\hline & 1 & 6 & 080 & 12079.37 \\
\hline & 4 & 3 & 080 & 13471.77 \\
\hline
\end{tabular}




\begin{tabular}{rrrrr}
6 & 6 & 1 & 080 & 14348.09 \\
6 & 6 & 0 & 080 & 14348.09 \\
7 & 0 & 7 & 080 & 11889.98 \\
8 & 1 & 8 & 080 & $12367.67^{a}$ \\
9 & 0 & 9 & 080 & 12284.81 \\
11 & 0 & 11 & 080 & 12789.28 \\
11 & 1 & 10 & 080 & 13444.72 \\
& & & & \\
0 & 0 & 0 & 090 & $12533.72^{b}$ \\
1 & 1 & 0 & 090 & $13060.42^{b}$ \\
2 & 2 & 1 & 090 & 13628.63 \\
3 & 3 & 0 & 090 & 14211.96 \\
4 & 1 & 4 & 090 & $13234.76^{b}$ \\
4 & 4 & 1 & 090 & 14805.74 \\
5 & 0 & 5 & 090 & 12880.60 \\
5 & 5 & 1 & 090 & 15412.32 \\
5 & 5 & 0 & 090 & 15412.32 \\
6 & 1 & 6 & 090 & 13461.76 \\
8 & 1 & 8 & 090 & 13781.23 \\
9 & 0 & 9 & 090 & 13611.87 \\
10 & 1 & 10 & 090 & 14203.64 \\
11 & 0 & 11 & 090 & 14162.66 \\
\hline & & & &
\end{tabular}

${ }^{a}$ Level determined with combination differences.

${ }^{b}$ Level determined from sunspot spectrum.

\section{CONCLUSIONS}

The technique of using variational nuclear motion calculations to fit iteratively experimentally determined energy levels to aid in the assignment of transitions 
of higher lying energy levels has been used previously to analyse spectra of hot water [15, 24]. Here we adapt this technique to the specific problem of identifying transitions between states with high levels of bending excitation because the bending potential near the region where water approaches linear geometries is poorly characterised by previous experimental studies [12]. Our fitted potential gives the best currently available empirical estimate of the barrier to linearity in water of $11114 \pm 5 \mathrm{~cm}^{-1}$, which is in very good agreement with the current best $a b$ initio value of $11119 \pm 15 \mathrm{~cm}^{-1}$ [13] and improves on the previous best empirical value of $11105 \pm 5 \mathrm{~cm}^{-1}$ [12] because of the extra data now available for the higher bending states.

This potential has allowed us to assign 134 new energy levels for states with 5 or more quanta of bending excitation. In particular we have obtained energy levels for the $9 \nu_{2}$ bending excited state of water for the first time and the first empirical values for the $7 \nu_{2}, 8 \nu_{2}$ and $9 \nu_{2}$ band origins. Particular emphasis was placed on assigning states with $J=K_{a}$, since they are the key states for identifying changing behaviour; these states are generally harder to identify that states with low $K_{a}$ which lie at lower energies and therefore are associated with higher intensity transitions. Appropriate plots of energy levels from these vibrational states show the clear change in behaviour predicted by Child et al. [4] on theoretical grounds. Our analysis strongly suggests that the bending levels of water behave as if there is a monodromy point close to the $7 \nu_{2}$ band origin at $10086 \mathrm{~cm}^{-1}$.

\section{Acknowledgements}

This work was supported by The Royal Society, INTAS, the UK Engineering and Physical Science Research Council, the Fonds National de la Recherche Scientifique (F.N.R.S., Belgium, F.R.F.C. convention No 2.4536.01), the "Actions de Recherches Concertés" (Communauté française de Belgique), the NASA astrophysics program, the Canadian Natural Sciences and Engineering Research Council and the Russian Fund for Fundamental Studies. OLP is grateful to the Dr. Barbara Mez-Starck Foundation for financial support.

\section{References}

[1] R. Cushman, Centruumvoor Wiskund Inf. Newslett. 1 (1983) 4.

[2] L. R. Bates, J. Appl. Math. Phys. (ZAMP) 42 (1991) 837.

[3] R. Cushman, B. Zhilinskii, J. Phys. A 35 (2002) L415.

[4] M. S. Child, T. Weston, J. Tennyson, Mol. Phys. 96 (1999) 371.

[5] M. P. Jacobson, M. S. Child, J. Chem. Phys. 114 (2001) 262. 
[6] M. Joyeux, D. A. Sadov, J. Tennyson, Chem. Phys. Lett 382 (2003) 439.

[7] B. P. Winnewisser, in Molecular Spectroscopy: Modern Research, vol. III, (1985) edited by K.N. Rao, Academic Press, Orlando, FL, p.321.

[8] B. P. Winnewisser, M. Winnewisser, I. R. Medvedev, M. Behnke, F. C. De Lucia, S. C. Ross, J. Kapot, Paper TH07, 60th International Symposium on Molecular Spectroscopy, Ohio State University, Columbus, OH, June 20-24, 2005; and in preparation for publication.

[9] K. Efstathiou, M. Joyeux, D. A. Sadovskii, Phys. Rev. A 69 (2004) 032504 .

[10] A. G. Császár, W. D. Allen, H. F. Schaefer III, J. Chem. Phys. 108 (1998) 9751.

[11] G. Tarczay, A. G. Császár, W. Klopper, V. Szalay, W. D. Allen, H. F. Schaefer III, J. Chem. Phys. 110 (1999) 11971.

[12] J. S. Kain, O. L. Polyansky, J. Tennyson, Chem. Phys. Lett. 317 (2000) 365.

[13] E. F. Valeev, A. G. Császár, W. C. Allen, H. F. Schaefer III, J. Chem. Phys. 114 (2001) 2875.

[14] H. Partridge, D. W. Schwenke, J. Chem. Phys. 106 (1997) 4618.

[15] P.-F. Coheur, P. F. Bernath, M. Carleer, R. Colin, O. L. Polyansky, N. F. Zobov, S. V. Shirin, R. J. Barber, J. Tennyson, J. Chem. Phys. 122 (2005) 074307.

[16] L. Wallace, P. F. Bernath, W. Livingston, K. Hinkle, J. R. Busler, B. Guo, K. Q. Zhang, Science 268 (1995) 1155.

[17] O. L. Polyansky, N. F. Zobov, S. Viti, J. Tennyson, P. F. Bernath, L. Wallace, Science 277 (1997) 346.

[18] O. L. Polyansky, N. F. Zobov, S. Viti, J. Tennyson, P. F. Bernath, L. Wallace, J. Mol. Spectrosc. 186 (1997) 422.

[19] O. L. Polyansky, N. F. Zobov, J. Tennyson, J. A. Lotoski, P. F. Bernath, J. Mol. Spectrosc. 184 (1997) 35.

[20] N. F. Zobov, O. L. Polyansky, J. Tennyson, J. A. Lotoski, P. Colarusso, K.-Q. Zhang, P. F. Bernath, J. Mol. Spectrosc. 193 (1999) 118.

[21] R. Lanquetin, L. H. Coudert, and C. Camy-Peyret, J Mol. Spectrosc. 195 (1999) 54.

[22] O. L. Polyansky, A. G. Császár, S. V. Shirin, N. F. Zobov, P. Barletta, J. Tennyson, D. W. Schwenke, P. J. Knowles, Science 299 (2003) 539.

[23] S. V. Shirin, O. L. Polyansky, N. F. Zobov, P. Barletta, J. Tennyson, J. Chem. Phys. 118 (2003) 2124.

[24] S. V. Shirin, N. F. Zobov, O. L. Polyansky, J. Tennyson, T. Parekunnel and P. F. Bernath, J. Chem. Phys., 120 (2004) 206.

[25] J. Tennyson, N. F. Zobov, R. Williamson, O. L. Polyansky and P. F. Bernath, J. Phys. Chem. Ref. Data, 30 (2001) 735. 


\section{Figure caption}

Figure 1. Experimental energy levels of $\mathrm{H}_{2}{ }^{16} \mathrm{O}$ for bending states with $J=K_{a}$. States are plotted for both positive and negative $J$ to emphasise the change in behaviour about the level with $J=0$ as the degree of bending excitation is increased. 